\title{
Inserção social nos Programas de Pós-graduação na área de Serviço Social
}

\section{Social insertion in Graduate Programs in the Social Work area}

\author{
Denise Bomtempo Birche de Carvalho ${ }^{a}$ \\ (D) https://orcid.org/0000-0001-6808-9660 \\ Inez Stampa ${ }^{b}$ \\ (D) https://orcid.org/0000-0003-3770-1129 \\ Joana Valente Santanac \\ (D https://orcid.org/0000-0003-4033-1317
}

Resumo: O artigo busca contribuir com as discussões sobre a relação universidade e sociedade, abordando a inserção social dos programas de pós-graduação stricto sensu na área de Serviço Social. Apresenta breve histórico sobre a inserção social como critério de avaliação pela Capes, além de panorama da inserção social como subsídio para a produção de conhecimento e como dimensão que reflete o compromisso ético, político, profissional e social, com problemas da sociedade em nível local, regional e nacional.

Palavras-chave: Inserção social. Pós-graduação. Serviço Social. Universidade e sociedade.

\begin{abstract}
The article seeks to contribute to the discussions on the relationship between university and society, addressing the social insertion of stricto sensu graduate programs in the area of Social Work. It presents a brief history of social insertion as a criterion for evaluation by Capes, in addition to a panorama of social insertion as a subsidy for the production of knowledge and as a dimension that reflects the ethical, political, professional and social commitment to society's problems at the local level, regional and national.
\end{abstract}

Keywords: Social insertion. Graduate programs. Social Work. University and society.

aPrograma de Pós-graduação em Política Social da Universidade de Brasília (UnB), Brasília/DF, Brasil.

'Programa de Pós-graduação em Serviço Social da Pontifícia Universidade Católica do Rio de Janeiro (PUC-Rio), Rio de Janeiro, RJ, Brasil.

'Programa de Pós-graduação em Serviço Social da Universidade Federal do Pará, Belém/PA, Brasil.

Recebido: 3/3/2020 - Aprovado: 25/5/2020 


\section{Introdução}

ste artigo discute, com base nos critérios de avaliação da Comissão de Aperfeiçoamento de Pessoal de Nível Superior (Capes), a questão da inserção social no processo avaliativo dos programas de pós-graduação (PPG) no Brasil. Seu propósito é contribuir com as discussões sobre a relação entre universidade e sociedade mediante a inserção social dos PPG stricto sensu (mestrado acadêmico e doutorado) na área do Serviço Social.

A pós-graduação, no Brasil, foi estruturada a partir do Parecer $n^{\circ}$ 977, de 3 de dezembro de 1965, exarado pela Câmara do Ensino Superior do Conselho Federal de Educação (CES/CFE). ${ }^{1}$ Com a Reforma Universitária de 1968, ficou definido o papel das universidades e o reforço à pós-graduação, que deveria se expandir e consolidar-se com o objetivo precípuo de formar recursos humanos "de alto nível, a pesquisa e o desenvolvimento das ciências, sobretudo em termos de novas tecnologias e conhecimento aplicado para o crescimento econômico do país" (Alves e Oliveira, 2014, p. 360).

No Brasil, o Sistema Nacional de Pós-graduação (SNPG) é coordenado pela Capes e tem como objetivos: certificar a qualidade da pós-graduação brasileira; ser referência para a distribuição de bolsas, auxílios e recursos para o fomento; identificar assimetrias regionais; identificar áreas estratégicas necessárias ao desenvolvimento do país e orientar ações de indução na criação e expansão de PPG no Brasil (Capes, 2020a). Nesse contexto, a Capes define os critérios de avaliação de desempenho dos PPG e inclui, entre eles, o item inserção social, objeto de discussão deste artigo.

$O$ texto contém, além da introdução e das considerações finais, mais três seções: a primeira discute a inserção social como quesito de avaliação da pós-graduação; a segunda discorre sobre inserção, relevância

1 Também conhecido como "Parecer Sucupira", do extinto Conselho Federal de Educação, hoje Conselho Nacional de Educação (CNE). 
e impacto social e econômico da pós-graduação, considerando o novo modelo de avaliação da Capes; a terceira trata da relevância e do impacto da pós-graduação, também nos aspectos social e econômico, para a área do Serviço Social.

\section{Inserção social como quesito de avaliação da pós-graduação}

Historicamente, o SNPG foi estruturado, institucionalizado e cresceu ao longo de mais de meio século. Atualmente, em 2020, o sistema de avaliação da Capes conta com três colégios (humanidades; ciências da vida; ciências exatas, tecnológicas e multidisciplinar) e abarca 49 áreas de conhecimento, com um total de 147 coordenadores (98 coordenadores acadêmicos, entre titulares e adjuntos, e 49 profissionais) (Capes, 2020a).

No âmbito da Capes, a avaliação da pós-graduação foi implantada em 1976 (Castro, 2006; Capes, 2020a). De 1976 a 1997, tinha periodicidade anual, sendo os cursos de mestrado e de doutorado avaliados separadamente, em uma escala de cinco conceitos (de A a E, considerando-se A o conceito mais alto). A Capes deu início à avaliação trienal, no período 1998-2000, com os seguintes quesitos de avaliação: Proposta do programa; Corpo docente; Atividade de pesquisa; Atividade de formação; Corpo discente; Teses e dissertações e Produção intelectual (Capes, 2001). A partir de 2013, a avaliação tornou-se quadrienal.

Considerando as mudanças de aperfeiçoamento do sistema avaliativo, incluiu-se, na Trienal de 2004-06 (realizada em 2007), o item inserção social na nova ficha de avaliação, que passou, assim, a ter cinco quesitos: (1) Proposta do programa; (2) Corpo docente; (3) Corpo discente, Teses e dissertações; (4) Produção intelectual e (5) Inserção social. ${ }^{2}$ Renato Janine

2 O quesito inserção social, por ser novo, teve seu peso definido pelo CTC-ES em 10\%, a ser aplicado por todas as áreas. Cf. Ribeiro (2007). 
Ribeiro, então diretor de avaliação da agência de fomento, afirma que cada área de conhecimento teria autonomia para definir "como entendia a inserção social", adotando como base "quatro exemplos de impacto e as indicações que a Capes formulou a respeito de cada um deles" (Ribeiro, 2007, p. 2-3), como segue:

1) Impacto tecnológico/econômico: contribuição para o desenvolvimento microrregional, regional e/ou nacional, destacando os avanços produtivos gerados; aumento da produtividade; disseminação de técnicas e conhecimentos que melhorem o desempenho econômico, respeitando e considerando seus efeitos sociais e ambientais;

2) Impacto educacional: contribuição para a melhoria do ensino básico, médio, graduação, técnico/profissional e para o desenvolvimento de propostas inovadoras de ensino [...];

3) Impacto propriamente social: formação de recursos humanos qualificados para a administração pública ou a sociedade civil que possam contribuir para o aprimoramento da gestão pública e a redução da dívida social, ou para a formação de um público que faça uso dos recursos da ciência e do conhecimento;

4) Impacto cultural: formação de recursos humanos qualificados para o desenvolvimento cultural e artístico, formulando políticas culturais e ampliando o acesso à cultura e às artes e ao conhecimento nesse campo.

Nossa posição sugere que a inserção social deve qualificar a relevância econômica, ético-política, social e cultural da pós-graduação em formar recursos humanos e produzir conhecimentos para responder aos problemas do país. Isso requer que o processo de planejamento e autoavaliação das instituições de ensino de pós-graduação estejam bem articulados às necessidades da sociedade, isto é, articulados à realidade local, regional e nacional. Mello, Almeida Filho e Ribeiro (2013, p. 5) afirmam que a inserção social poderia ser considerada "macroextensão", pois "envolve ações de inserção ou de impacto social que obedecem a uma estratégia e afetam globalmente o campo de ação conexo àquela área de conhecimento". Nessa perspectiva, os indicadores de inserção 
social devem estar articulados aos objetivos da pós-graduação de um modo geral e, em particular, à natureza da área de conhecimento, à organicidade entre área de concentração, linhas e projetos de pesquisa, às temáticas trabalhadas com os alunos e à inserção dos egressos no mercado de trabalho.

\section{Inserção, relevância e impacto social e econômico da pós-graduação no novo modelo de avaliação}

Em dezembro de 2016, a Capes instituiu a Comissão Especial de Acompanhamento do PNPG 2011-2020, ${ }^{3}$ a qual demandou diagnóstico e recomendações sobre o SNPG após os primeiros cinco anos de vigência do referido plano, sendo que a comissão deveria apresentar projeções e recomendações para o quinquênio 2016-20. Para tanto, a comissão solicitou, em novembro de 2017, contribuições e propostas de setores e entidades ${ }^{4}$ sobre mudanças que seriam necessárias, principalmente em relação ao modelo vigente de avaliação da pós-graduação stricto sensu (Capes, 2018b).

O relatório final da comissão apontou, conforme a seguir elencados, os pontos convergentes e recorrentes no processo de avaliação da pós-graduação brasileira: autoavaliação dos PPG como critério de avaliação, inclusive vinculando-a ao Plano de Desenvolvimento Institucional (PDI) das IES; impacto dos PPG (no desenvolvimento social e econômico, tanto regional como nacional); modelo único de avaliação

3 Portaria no 203, de 14 de novembro de 2016, com representantes da Capes, PUCRS, Unifesp, UFR, Unicamp, Foprop, UFS, UFRGS, CNE, PQTEC/SJCヌSP, ANPG. Disponível em: http://www. capes.gov.br/plano-nacional-de-pos-graduacao. Acesso em: 10 set. 2019.

4 A Comissão Especial foi composta por membros da comunidade acadêmica oriundos das seguintes entidades: ABC, Andifes, Abruem, Abruc, CNE, Confap, Consecti, CNPq, CTC-ES, Finep, Foprop, MCTIC, MDIC, SBPC, SBC, Anped. As contribuições das entidades podem ser visualizadas em:www.capes.gov.br/images/novo_portal/documentos/PNPG/2018_PNPG_CS_Avaliacao_Final_10_10_18_CS_FINAL_17_55.pdf. Acesso em: 15 jan. 2020. 
(multidimensional); produções científicas (bibliográficas e técnicas indicadas); relevância social e econômica dos PPG; acompanhamento de egressos; equilíbrio entre indicadores quantitativos e qualitativos; mudanças no Qualis, principalmente no Qualis periódicos; internacionalização e inovação (Capes, 2018b). Com base nesses pontos, o Conselho Técnico-Científico da Educação Superior da Capes (CTC-ES) criou grupos de trabalho (GT) específicos ${ }^{5}$ com o objetivo de propor as mudanças requeridas para o aperfeiçoamento do processo e dos instrumentos de avaliação da pós-graduação. São eles: Autoavaliação dos Programas de Pós-Graduação; Ficha de Avaliação; Produção Técnica; Proposta de Classificação de Livros; Qualis Artístico e Classificação de Eventos; Critérios Avaliativos para Propostas de Cursos Novos na Modalidade EAD; Impacto e Relevância Econômica e Social; Internacionalização; Inovação e Transferência de Conhecimento; Qualis Periódicos (Capes, 2018b, p. 10).

Não é nosso objetivo, neste artigo, discorrer sobre as propostas de cada um dos GT. Vamos, portanto, nos ater ao GT Impacto e Relevância Econômica e Social (doravante apenas GT), pois este é o grupo que se ocupa da inserção social da pós-graduação e de seus impactos na sociedade, tendo como objetivo definir o escopo da dimensão desse impacto e a relevância social e econômica dessa inserção no processo de avaliação dos PPG. Trata-se de "definir de maneira uniforme para todas as áreas de avaliação da Capes conceitos, variáveis e indicadores que representem o tema" (Capes, 2020b).

Deve-se ressaltar que a ficha de avaliação para o quadriênio 201720, em paralelo às discussões sobre os GTS, foi redimensionada de cinco para apenas três quesitos: (1) Programa, (2) Formação e (3) Impacto na sociedade, ${ }^{6}$ sendo que a inserção social está incluída nesse último

5 Disponível em: http://capes.gov.br/relatorios-tecnicos-dav. Acesso em: 15 jan. 2020. Note-se que na página da Capes não está elencado o GT Qualis Periódicos, o qual foi acrescido aos grupos pelas autoras.

6 Ver a descrição desses três quesitos disponível em: http://capes.gov.br/relatorios-tecnicos-dav. Acesso em: 15 jan. 2020. 
quesito, devendo ser valorizada e aferida por "meio da pesquisa aplicada, cujos resultados possam contribuir para a melhoria da qualidade de vida da população e gerem ainda impacto em segmentos da sociedade, mercados ou organizações" (Capes, 2018b, p. 18). No entanto, para que a pós-graduação possa gerar impacto na sociedade ela precisa satisfazer duas condições de forma simultânea, de acordo com a concepção do GT: "deve ser percebida como algo relevante e solucionar problemas econômicos, sociais, ambientais, culturais, tecnológicos, dentre outros; apresentar resultados, sob a forma de ideias, produtos e serviços, que respondam às demandas da sociedade, do Estado e do mercado" (Capes, 2020b, p. 15).

Para configurar a natureza do impacto econômico e social da pós-graduação, foi utilizada a classificação realizada pelo GT Produção Técnica (Capes, 2020c), que já havia elaborado um questionário contendo 21 produtos técnico-tecnológicos, aos quais foram acrescidos dois, resultando uma lista de 23 produtos $^{7}$ (Capes, 2020d, p. 17).

Como resultado, os estudos do GT apontam os principais indicadores a ser empregados na avaliação da inserção, da relevância e do impacto dos PPG na sociedade: "causalidade; tipo de impacto; duração prevista; classe do impacto; setor beneficiado; vínculo com o PDI, tipo de aplicação, disponibilidade, setor beneficiado e abrangência territorial" (Capes, 2020b, p. 42). Contudo, acrescenta o GT, em suas conclusões, que cada área de conhecimento deverá elaborar seus próprios indicadores de relevância e de impacto, de acordo com sua natureza, seus produtos (bibliográficos e técnicos) e sua relação com a sociedade (Capes, 2020b).

1. Produto bibliográfico; 2. Ativos de propriedade intelectual; 3. Tecnologia social; 4. Curso de formação profissional; 5. Produto de editoração; 6. Material didático; 7. Software/aplicativo; 8. Evento organizado; 9. Norma ou marco regulatório; 10. Relatório técnico conclusivo; 11. Manual/protocolo; 12. Tradução; 13. Acervo; 14. Base de dados técnico-científica; 15. Cultivar; 16. Produção de comunicação; 17. Carta, mapa ou similar; 18. Produtos/processos em sigilo; 19. Taxonomia, ontologias e tesauros; 20. Empresa ou organização social inovadora; 21. Processo, tecnologia, e produto não patenteável; 22. Proposição de iniciativa legislativa ou plano de ação, política ou ação governamental; 23. Serviços (Capes, 2020d). 


\section{Inserção, relevância e impacto social e econômico da pós-graduação para a área do Serviço Social}

O relatório do GT Impacto e Relevância Econômica e Social apontou que "um critério fundamental para avaliar as repercussões sociais dos cursos seria sua participação/aderência contínua (e não apenas em ações pontuais) em políticas públicas" (Capes, 2018a, p. 18). No documento de área do Serviço Social de 2009, foi evidenciado que o quesito inserção social tem grande importância, dada à natureza da área (de conhecimento e de intervenção na realidade) e de sua vinculação com a formulação, a implementação e a avaliação de políticas públicas (Capes, 2009). 0 relatório de avaliação da área de Serviço Social (2007-09) revelou que as atividades desenvolvidas pelos PPG da área estão em sintonia "com os desafios que se colocam ao desenvolvimento social do país, que se faz acompanhar de profundas desigualdades" (Capes, 2010, p. 20).

Nessa perspectiva, o Serviço Social volta-se para a análise da questão social e de suas manifestações mais contundentes, no marco das relações entre o Estado e a sociedade, tal como se expressam na vida cotidiana de distintos segmentos das classes subalternas, em suas relações com o bloco no poder e com as iniciativas coletivas pela conquista, efetivação e ampliação dos direitos sociais.

Foi com base na posição acadêmica - ética, política e profissional - e nas premissas supracitadas que a área de Serviço social respondeu o questionário do GT Produção Técnica, e, dentre os 23 produtos selecionados por esse grupo, a área de Serviço Social escolheu os dez que mais caracterizam a inserção social, a relevância e o impacto social, em seus aspectos econômico, educacional, ambiental e cultural da área, em nível local, regional, nacional e internacional. São eles: Produto bibliográfico; Tecnologia social; Curso de formação profissional; Produto de editoração; Material didático; Evento organizado; Relatório técnico conclusivo; Base de dados técnico-científica; Proposição de iniciativa legislativa ou de plano, política ou ação governamental; Serviços. 
Para cada produto selecionado deveriam ser indicados os principais mecanismos de transferência de conhecimento dos PPG da área de Serviço Social para a sociedade, que ficaram assim formulados: cursos, assessorias e capacitações de profissionais de Serviço Social e área afins, com vistas a aprimorar o planejamento, elaborar diagnósticos, execução e avaliação de programas governamentais e não governamentais, nos diversos campos de políticas públicas e nos segmentos sociais de atuação do assistente social, além das assessorias e das capacitações decorrentes de supervisão de estágio curricular obrigatório, nos cursos de graduação em Serviço Social.

Para efeito deste artigo, foi realizado um levantamento em relatórios de 33 PPG da área de Serviço Social, disponíveis na Plataforma Sucupira, referente aos anos de 2017 e 2018, com o objetivo de sistematizar (ainda que de forma breve) os registros das atividades realizadas pelos programas no item inserção social.

Nesse item, todos os PPG da área de Serviço Social registram atividades de assessoria e consultoria para formulação, gestão, implementação, análise e avaliação de políticas públicas, bem como atividades de extensão universitária, claramente em defesa dos direitos da classe trabalhadora, sendo que as referidas atividades de assessoria são realizadas nas áreas de: assistência social, com destaque para a participação de docentes no Programa Nacional de Capacitação do Suas/CapacitaSuas); saúde; educação; crianças e adolescentes; gênero/ atenção aos direitos das mulheres; direitos humanos; direito à cidade; questões sobre trabalho; direitos da população LGBT; questão racial e indígena; segurança alimentar, questão de fronteiras/migrações; território, dentre outras.

Os referidos PPG assinalam como importante a articulação com a Associação Brasileira de Ensino e Pesquisa em Serviço Social (Abepss), com o Conselho Federal de Serviço Social (CFESS) e com os Conselhos Regionais de Serviço Social (Cress), mediante participação em diretorias ou como membro de comissões/grupos temáticos de pesquisa. 
As atividades registradas nos relatórios dos PPG da área de Serviço Social demonstram que a área dialoga com as orientações da Capes no que se refere à inserção social, considerando que essas atividades voltam-se para a melhoria da qualidade de vida da população brasileira, com potencialidade de geração de impactos na sociedade, especialmente em segmentos da classe trabalhadora.

\section{Considerações finais}

O Serviço Social se configura como área de conhecimento e como profissão. No Brasil, sua regulamentação data de agosto de 1953, destacando-se, desde essa época, três competências profissionais: (i) atuar no âmbito das políticas públicas e privadas, no planejamento, na gestão, no monitoramento, na avaliação de programas e de serviços sociais, e no trabalho de advocacy com os movimentos sociais; (ii) planejar, executar e avaliar pesquisas que possam contribuir para a análise da realidade social e para o subsídio de ações profissionais; e (iii) formar recursos humanos para produzir conhecimento científico crítico sobre a realidade social e sobre a própria ação profissional (Capes, 2019).

Nessa perspectiva, suas produções científicas (bibliográficas e técnicas) possuem relevância e impacto sobre os processos formativos, a divulgação do conhecimento, a formulação, execução e avaliação de políticas públicas e a organização de serviços sociais, contribuindo, ainda, para a formulação de legislações de políticas e serviços. Ademais, a produção intelectual da área do Serviço Social vem contribuindo não só para o conhecimento crítico das diversas expressões da questão social e das desigualdades sociais, nos âmbitos local, regional, nacional e internacional, em especial na América Latina, mas também para a proposição de respostas ao seu enfrentamento. 


\section{Referências}

ALVES, M. F.; OLIVEIRA, J. F. Pós-graduação no Brasil: do regime militar aos dias atuais. Revista Brasileira de Política e Administração da Educação - RBPAE, v. 30, n. 2, p. 351-376, maio/ago. 2014.

CAPES. Avaliação trienal 2001. Disponível em: https:/www.capes.gov.br/avaliacao/ permanencia-no-snpg-avaliacao/avaliacoes-anteriores/91-conteudo-estatico/avaliacaocapes/6821-avaliacao-trienal-2001. Acesso em: 15 jan. 2020.

CAPES. Documento de área de Serviço Social 2009. Disponível em: http://capes.gov. br/images/stories/download/avaliacao/SERVICOSOCIAL_22jun10b.pdf. Acesso em: 6 jan. 2020.

CAPES. Relatório de avaliação da Área de Serviço Social - 2007/2009, Trienal de 2010. Disponível em: http://trienal.capes.gov.br/wp-content/uploads/2011/01/SERVIÇO-SOCIALRELATÓRIO-DE-AVALIAÇÃO-FINAL-jan11.pdf. Acesso em: 6 jan. 2020.

CAPES. Plano Nacional de Pós-graduação. 2018a. Disponível em: http://www.capes.gov. br/plano-nacional-de-pos-graduacao. Acesso em: 10 set. 2019.

CAPES. Proposta de aprimoramento do modelo de avaliação da PG. 2018b. Disponível em: www.capes.gov.br/images/novo_portal/documentos/PNPG/2018_PNPG_CS_Avaliacao_ Final_10_10_18_CS_FINAL_17_55.pdf. Acesso em: 15 jan. 2020.

CAPES. Documento de área Serviço Social 2019. Disponível em: http://capes.gov.br/images/ Documento_de_área_2019/DOC_SERVICO_SOCIAL_01_11.pdf. Acesso em: 15 dez. 2019.

CAPES. Caracterização do sistema de avaliação da pós-graduação. 2020a. Disponível em: http://www.capes.gov.br/acessoainformacao/informacoes-classificadas/91-conteudoestatico/avaliacao-capes/6871-caracterizacao-do-sistema-de-avaliacao-da-posgraduacao. Acesso em: 5 jan. 2020.

CAPES. Relatórios técnicos da Diretoria de Avaliação. 2020b. Disponível em: http://capes. gov.br/relatorios-tecnicos-dav. Acesso em: 15 jan. 2020.

CAPES. Relatório do GT Produção Técnica. 2020c. Disponível em: http://capes.gov.br/ images/novo_portal/documentos/DAV/avaliacao/10062019_Produção-Técnica.pdf. Acesso em: 9 jan. 2020.

CAPES. Relatório do GT Impacto e Relevância Econômica e Social. 2020d. Disponível em: chrome- http://capes.gov.br/images/RELATORIOS_GTS/2020-01-03_Relatório_GTImpacto-e-Relevancia-Economica-e-Social.pdf. Acesso em: 14 jan. 2020.

CASTRO, C. C. A CAPES na visão de ex-diretor. Análise, Porto Alegre, v. 17, n. 2, p. 360-376, jul./dez. 2006. 
MELLO, A. F.; ALMEIDA FILHO, N.; RIBEIRO, R. J. Por uma universidade socialmente relevante. 2013. Disponível em: http://portal.mec.gov.br/dmdocuments/cne_alexfiuza. pdf. Acesso em: 6 jan. 2020.

RIBEIRO, R. J. Inserção social. 2007. Disponível em: https:/www.capes.gov.br/avaliacao/ permanencia-no-snpg-avaliacao/avaliacoes-anteriores/91-conteudo-estatico/avaliacaocapes/6878-artigos. Acesso em: 6 jan. 2020.

\section{Sobre as autoras}

Denise Bomtempo Birche de Carvalho - Professora titular do Departamento de Serviço Social e professora do Programa de Pós-graduação em Política Social; professora emérita da Universidade de Brasília; coordenadora da área de Serviço Social na Capes (2018-22).

E-mail: denisebomtempo@unb.br, denisebomtempo@terra.com.br

InEz STAMPA - Professora do Departamento e do Programa de Pós-graduação em Serviço Social; coordenadora adjunta da área de Serviço Social na Capes (2018-22). E-mail: inezstampa@gmail.com

Joana Valente Santana - Doutora em Serviço Social pela UFRJ; docente da Faculdade e do Programa de Pós-graduação em Serviço Social da UFPA; coordenadora adjunta da área de Serviço Social na Capes (2018-22).

E-mail: joanavalente@ufpa.br, joanavalentesantana@gmail.com 